\begin{tabular}{c} 
International Journal of Scientific World, $4(2)(2016) 40-42$ \\
International Journal of Scientific World \\
SPC \\
Website: $\begin{array}{c}\text { ww. sciencepubco.com/index. } h \text { h } / I J S W \\
\text { doi: } 10.14419 / i j s w . v 4 i 2.6273 \\
\text { Research paper }\end{array}$ \\
\hline
\end{tabular}

\title{
Prevalence and antibiotic resistance of Pasteurella multocida isolated from chicken in Ado-Ekiti metropolis
}

\author{
Atere Ayowole Victor ${ }^{1 *}$, Bamikole Akinfolarin Mathew ${ }^{1}$, Oluyege Adekemi Olubukunola ${ }^{1}$, \\ Ajurojo Oluwaseun Ayo ${ }^{2}$, Alo Odunayo Samuel ${ }^{2}$ \\ ${ }^{1}$ Department of Microbiology, Ekiti State University, Nigeria \\ ${ }^{2}$ Metrovet Veterinary Hospital, Ado Ekiti, Nigeria \\ *Corresponding author_E-mail: victor_efosa001@yahoo.com
}

\begin{abstract}
Pasteurella multocida is a poultry bacterial pathogen causing fowl cholera in chicken. The prevalence and antibiotic susceptibility of $P$. multocida isolates from freshly dead chicken were determined. Ninety seven (97) freshly dead chicken from 23 different farms were analyzed for the presence of $P$. multocida. Swabs of the trachea and the liver of the necropsied chicken were activated on buffered peptone water and later cultured on blood agar and MacConkey agar. Pure culture of organisms were subjected to cultural and biochemical characterization. In vitro susceptibility of the pure isolates of $P$. multocida against 12 antimicrobial agents was determined using disk diffusion method. Twelve isolates of $P$. multocida were recovered from the chicken, with a prevalence of $12.4 \%$. Nine of the isolates were recovered from the trachea and three from the liver. All the 12 isolates recovered from the birds were multi-resistant to the antibiotics used in this research. The antibiogram showed that all the isolates resisted ampicillin, amoxicillin/clavulinate, doxycycline and tylosine. Nitrofuratoin and gentamycin had the best antimicrobial activity with $25 \%$ and $50 \%$ resistance respectively. The resistance of other antibiotics are: Ofloxacin 75\%, Ciprofloxacin 83.3\%, Enrofloxacin 75\%, Furasol 66.7\%, Ceftazidime 91.7\% and Cefuroxime 66.7\%. This result showed that there is an emergence of multi- resistance in P. multocida, therefore it is important to carry out sensitivity test before administration of antibiotics in order to control fowl cholera.
\end{abstract}

Keywords: Antibiotics; Fowl Cholera; Pasteurella multocida; Resistance.

\section{Introduction}

Fowl cholera is a highly contagious disease caused by Pasteurella multocida. This organism affects a broad host range of birds both domestic and wild birds. It causes high mortality that incur significant economic losses in commercial and backyard poultry production [15]. It spreads by contamination of feed or water, by ora or nasal discharges from infected birds. Migratory birds have been reported as a major source of fowl cholera [8].

Although, vaccines are given to birds against fowl cholera, yet fowl cholera has remain one of the main causes of loss in poultry [12]. P. multocida is also responsible for atrophic rhinitis in swine, snuffles in rabbit, septicaemia haemorhagica ovis in goat, pneumonia in cattle and haemorhagic septicaemia in cattle and buffalo, showing that $P$. multocida is not host specific [1], [18]. Antibiotics are used to a large extent for the treatment of fowl cholera. However, prolong and pervasive use of antibiotics has resulted in $P$. multocida acquiring resistance to most of the commonly used antimicrobials [2].

Antibiotic resistance of $P$. multocida isolates varies according to the host animal, specie, time, geographical origin and antimicrobial pre-treatment of the animal [7]. Multi-resistance pathogenic bacteria in food-producing animals and environmental sources is recognized as a global problem for public health [6], [21]. Multiple antibiotics are often recommended for the treatment of fowl cholera in Nigeria [10]. However, there is little information about multiple drug resistance of $P$. multocida as well as the prevalence of the pathogen in poultry; therefore this research is to document the results of multi-resistance $P$. multocida in Ekiti States, South Western Nigeria.

\section{Methodology}

Samples were collected from twenty three farms between January and June 2015, transported to the microbiology laboratory within two hours. The samples (ninety seven freshly dead chicken) were necropsied, swabs were collected aseptically from the trachea and the liver for bacteria isolation.

\subsection{Bacteriology}

The swabs collected from both the liver and the trachea were activated in buffered peptone water for 5 hours at $37^{\circ} \mathrm{C}$. A loop full of the activated organisms in the buffered peptone water were inoculated onto MacConkey agar (Biomark) and sheep blood agar by streaking. The plates were incubated at $37^{\circ} \mathrm{C}$ for 24 hours in an incubator (Royalcare England. DNP 9022A). The appearance of a zone of erythrocyte lysis around or under bacterial colonies indicated hemolysis on sheep blood agar.

\subsection{Cultural and biochemical characterization}

Pasteurella multocida isolates were selected based on the cultural characteristics on blood agar. The morphological appearance was also determined. Further confirmation was done by biochemical 
tests some of which are: motility, catalase, oxidase, $\mathrm{H}_{2} \mathrm{~S}$ production, nitrate, urease, indole, methyl red, Voges-Proskauer and citrate use tests [9].

\subsection{Antimicrobial drug sensitivity test}

In vitro susceptibility of the identified Pasteurella multocida isolates against antimicrobial agents was determined by the standard disk diffusion procedure. The organisms were standardized using McFarland standard at the absorbance of $450 \mathrm{~nm}$. The samples were inoculated on Muller-Hinton agar. The following antimicrobial agents were tested: Ceftazidime (CAZ $30 \mu \mathrm{g})$, Cefuroxime (CRX $30 \mu \mathrm{g}$ ), Gentamicin (GEN $10 \mu \mathrm{g}$ ), Ciprofloxacin (CPR 5 $\mu \mathrm{g}$ ), Ofloxacin (OFL $5 \mu \mathrm{g}$ ), Nitrofurantoin (NIT $300 \mu \mathrm{g}$ ), Ampicillin (AMP $10 \mu \mathrm{g}$ ), Amoxicillin/Clavulinate (AUG $30 \mu \mathrm{g}$ ), Enrofloxacin (ENR10 $\mu \mathrm{g}$ ), Furasol (FUR $10 \mu \mathrm{g}$ ), Tylosin (TLY 10 $\mu \mathrm{g})$ and Doxycycline (DOX10 $\mu \mathrm{g}$ ). Following the application of antimicrobial discs, the plates were incubated at $37{ }^{\circ} \mathrm{C}$ for $24 \mathrm{~h}$ in an incubator (Royalcare England. DNP 9022A). The diameters of the zones of inhibition were measured (millimetres) and were compared to internationally accepted standard to determine the susceptibility or resistance of the isolate [17].

\section{Results}

Pasteurella multocida were detected in 12 of the 97 cases investigated with a prevalence of $12.4 \%$. Extended phenotypic and biochemical characterization confirmed the isolates as $P$. multocida. The $P$. multocida isolates produced small, round, grayish, smooth, mucoid glistening and dewdrop-like colonies on blood agar plates and were Gram-negative coccobacilli. The strains did not grow on McConkey agar and were non-haemolytic on blood agar. Biochemical testing showed that all strains were urease negative, oxidase, citrate, indole and catalase positive. All the strains fermented galactose, fructose, D-glucose, D-mannitol and sucrose, while no reaction was recorded for inositol, raffinose and salicin. The observation made on the samples revealed that most of the $P$. multocida isolated from the chicken were resistant to most group of antibiotic as shown in table 1 .

Table 1: Percentage (\%) Antibiotic Resistant of Pasteurella multocida Isolated from Chicken Trachea and Liver.

\begin{tabular}{|c|c|c|c|c|c|c|c|c|c|c|c|c|}
\hline Isolates & AMP & AUG & OFL & TLY & CPR & ENR & DOX & FUR & GEN & NIT & CAZ & CRX \\
\hline Trachea & 100 & 100 & 66.7 & 100 & 88.9 & 77.8 & 100 & 66.7 & 33.3 & 22.2 & 88.9 & 55.6 \\
\hline $\mathrm{n}=9$ & (9) & (9) & (6) & $(9)$ & (8) & (7) & (9) & (6) & (3) & (2) & $(8)$ & $(5)$ \\
\hline Liver & 100 & 100 & 100 & 100 & 66.7 & 66.7 & 100 & 66.7 & 100 & 33.3 & 100 & 100 \\
\hline Total & 100 & 100 & 75 & 100 & 83.3 & 75 & 100 & 66.7 & 50 & 25 & 91.7 & 66.7 \\
\hline $\mathrm{n}=12$ & (12) & (12) & (9) & (12) & (10) & (9) & (12) & $(8)$ & (6) & (3) & (11) & $(8)$ \\
\hline
\end{tabular}

Key: Ampicillin (AMP), Amoxicillin/Clavulinate (AUG), Ofloxacin (OFL), Tylosin (TLY), Ciprofloxacin (CPR), Enrofloxacin (ENR), Doxycycline (DOX), Furasol (FUR), Gentamicin (GEN), Nitrofurantoin (NIT), Ceftazidime (CAZ) and Cefuroxime (CRX).

Nitrofurantoin was found to be the most effective antibiotic with a resistance of (3) $25 \%$, while the organism form (12) $100 \%$ resistance to ampicillin, amoxicillin/clavulinate, doxycycline and tylosine. Table 2 showed the antibiotic resistance pattern of the $P$. multocida isolates.

Table 2: Antibiotic Resistance Pattern of P. Multocida Isolated from Chicken Trachea and Liver.

\begin{tabular}{lll}
\hline Antibiotics & No of isolates & Percentage resistance (\%) \\
\hline AMP, AUG, OFL, TLY, CPR, ENR, DOX, FUR & 4 & 33.3 \\
ENR, DOX, FUR, TYL & 7 & 58.3 \\
AMP, AUG, CRX, CAZ & 8 & 66.7 \\
GEN, CRX, NIT, CAZ & 1 & 8.3 \\
TYL, AMP, DOX, AUG & 12 & 100 \\
CAZ, CRX, GEN & 5 & 41.7 \\
FUR, ENR, CPR & 6 & 50 \\
NIT, OFL & 2 & 16.7 \\
\hline
\end{tabular}

Key: Ampicillin (AMP), Amoxicillin/Clavulinate (AUG), Ofloxacin (OFL), Tylosin (TLY), Ciprofloxacin (CPR), Enrofloxacin (ENR), Doxycycline (DOX), Furasol (FUR), Gentamicin (GEN), Nitrofurantoin (NIT), Ceftazidime (CAZ) and Cefuroxime (CRX).

\section{Discussion}

The occurrence of fowl cholera in commercial poultry birds had been reported as the major concern in the poultry industry by other workers [13], [14], and [16]. In this study, $12.4 \%$ isolation rate of $P$. multocida doubles the level reported by Mbuthia et al. [14] who recorded a rate of $6.2 \%$. This difference may be due to the number of samples, method of isolation, presence of stress and age of birds sampled. Isolation of $P$. multocida in the trachea and liver of chicken was earlier reported by Dashe et al. [10].

The antibiotic resistance of the P. multocida in this study showed a high resistance to the tested antibiotics compare to reports of previous studies; Everlon et al. [11] reported antibiotic resistance of P. multocida to range between 1.5 and $5.2 \%$ in isolates from chicken while Dashe et al. [10] reported resistance ranging between 6.7 and $46.7 \%$. In other various reports [2], [15], [20] it was found that the resistance level is not as high as found in this research. This may have been the result of misuse of antibiotics by most of the farmers in the territory even before they report to the clinic. Generally, farmers report cases in their farms after they have tried all the possible means, which often include heavy usage of antibiotics before making any clinical report.
The high resistance of $P$. multocida isolates to ampicillin, amoxicillin/clavulanate, doxycycline and tylosin has highlighted that prevention and therapeutic effect on avian $P$. multocida strains in Ekiti, Nigeria should no longer be expected from these antibiotics. The multidrug resistance of $P$. multocida is presumably attributed to the use of antibiotics as additives in poultry feed, extensive and pervasive use of antimicrobial agents by poultry farmers and Veterinary practitioners [4], [10]. Arora et al. [2] also recorded that injudicious use of antibiotics in poultry has contributed remarkably in the resistance of $P$. multocida. Another possible reason for the multiple resistance of $P$. multocida could be attributed to the proliferation of fake or sub-standard drug in Nigeria [10].

The result observed in the resistance of isolates of $P$. multocida from freshly dead chicken is similar to what was reported for Escherichia coli and Salmonella sp. Atere et al. [5] and Atere [3] reported a high resistant level in the E. coli and Salmonella isolates respectively, which was attributed to misuse of antibiotics before clinical reports. Antimicrobial resistance in P. multocida has been linked to small plasmids [11]. The coexistence and spread of these small plasmids has resulted in P. multocida isolates that are multi-resistant [19]. In this study, nitrofuratoin and gentamycin showed the highest sensitivity, this may have been based on the fact that these antibiotics are not readily used in poultry. 


\section{Conclusion}

With the increase in antibiotic resistance found in P. multocida, it is therefore recommended that antibiotic sensitivity test should be carried out before treatment; this will go a long way in the control of fowl cholera. It is also of great importance to encourage farmers to consult veterinary clinics (services), avoid self-prescription and carry out laboratory tests before any administration.

\section{References}

[1] Arashima, Y. and Kumasaka, K. (2005). Pasteurellosis as zoonosis. Med. 44 692-693 http://dx.doi.org/10.2169/internalmedicine.44.692.

[2] Arora, A. K., Virmani, S. K. J. and Oberoi, M. S. (2005). Isolation, characterization and antibiogram of Pasteurella multocida isolates from different animal species. Indian J. Anim. Sci., 75: 749-752.

[3] Atere, A. V. (2016). Multidrug resistant Salmonella sp isolated from chicken. International Journal of Biological research, 4 (1) 64 66.

[4] Atere, V. A, Bamikole, A. M. and Ajurojo, O. A. (2015) Antibiotic Susceptibility of Bacteria Isolated from Poultry Feeds Sold in Ado Ekiti, Nigeria. Journal of Advancement in Medical and Life Sciences V3I2.

[5] Atere, V. A., Bamikole, A. M., Ajurojo, O. A. and Alo, O. S. (2015). Antimicrobial Resistance Pattern of Pathogenic Escherichia coli Isolated from Chicken Liver and Trachea. Journal of Advancement in Medical and Life Sciences. V3I3.

[6] Bronzwaer, S. L., Cars, O., Buchholz, U. Molstad, S., Goettsch, W., Veldhuijzen, K. I., Kool, J. L., Sprenger, M. J. and Degener, J. E. (2002). European study on the relationship between antimicrobial use and antimicrobial resistance. Emerging Infectious Diseases, 8: 278-282. http://dx.doi.org/10.3201/eid0803.010192.

[7] Caprioli, A., L., Busani, J. L. and Helmuth, R. (2000). Monitoring of antibiotic resistance in bacteria of animal origin: Epidemiological and microbiological methodologies. Int. J. Antimicrobial Agents, 14: 295- 301. http://dx.doi.org/10.1016/S0924 8579(00)00140-0.

[8] Christensen, J. P., Dietz, H. H. and Bisgaard, M. (1998). Phenotypic and genotypic characters of isolates of Pasteurella multocida obtained from backyard poultry and from two outbreaks of avian cholera in avifauna in Denmark. Avian Pathology. 27: 373-381. http://dx.doi.org/10.1080/03079459808419354

[9] Cowan, S. T. and Steel, K. J. (1970). Manual for the Identification of Medical Bacteria. 1st ed. Cambridge University Press, London, UK.

[10] Dashe, Y. D., Raji, M. A., Abdu, P. A., Oladele, B. S. and Sugun, M. Y. (2013). Multidrug Resistant Pasteurella multocida Strains Isolated from Chickens with Cases of Fowl Cholera in Jos, Nigeria. Int. J. Poult. Sci., 12 (10): 596-600. http://dx.doi.org/10.3923/ijps.2013.596.600.

[11] Everlon, C. R., Patrick, J. B., Renato, P. M. and Fernando, A. Á (2013) Identification and antimicrobial susceptibility patterns of Pasteurella multocida isolated from chickens and Japanese quails in Brazil. Brazilian Journal of Microbiology 44 (1): 161-164. http://dx.doi.org/10.1590/S1517-83822013000100023.

[12] Glisson, J. R., Hofacre, C. L. and Christensen, J. P. (2003). Avian pasteurellosis. In: Saif, Y. M., Barnes, H. J., Glison, J. R., Fadly, A. M., McDougald, L. R. and Swayne, D. E. (Eds), Diseases of Poultry, 11th Ed. Iowa State University Press, Ames, Iowa, USA. Pp. 658-676.

[13] Kumar A. A., Shivachandra S. B., Biswas A., Singh V. P., Singh V. P. and Srivastava S. K. (2004). Prevalent serotypes of Pasteurella multocida isolated from different animal and avian species in India. Vet Res Comm, 28 (8): 657-667. http://dx.doi.org/10.1023/B:VERC.0000045959.36513.e9.

[14] Mbuthia G., Njagi L. W., Nyaga P. N., Bebora L. C., Minga U., Kamundia J. and Olsen J.E. 2008. Pasteurella multocida in scavenging family chickens and ducks: carrier status, age susceptibility and transmission between species. Avian Pathol, 37 (1): 51-57. http://dx.doi.org/10.1080/03079450701784891.

[15] Moemen, A. M., Mohamed-Wael, A. M., Ahmed, I. A., Awad, A. I. and Mohamed, S. A. (2012). Pasteurella multocida in backyard chickens in Upper Egypt: incidence with polymerase chain reaction analysis for capsule type, virulence in chicken embryos and antimicrobial resistance. Veterinaria Italiana, 48 (1): 77-86
[16] Muhairwa, A. P., Mtambo, M. M. A., Christensen, J. P. and Bisgaard, M. (2001). Occurrence of Pasteurella multocida and related species in village free ranging chickens and their animal contacts in Tanzania. Vet Microbiol, 78: 139-153. http://dx.doi.org/10.1016/S0378-1135(00)00296-0.

[17] Quinn, P. J., Carter, M. E., Markey, B. and Carter, G. R. (1994). Clinical veterinary Microbiology. Wolf, London, Pp. 95-102.

[18] Rimler, R. B. and Glisson, J. R. (1997). Fowl cholera. In: B.W. Calnek. H. J., Barnes, E.W. Beard., L.R. McDougald and Y.M. Saif (Eds.), Diseases of Poultry 10th Edn., Ames, Iowa State University Press, pp: 143-159.

[19] San Millan, A., Escudero, J. A., Gutierrez, B., Hidalgo, L., Garcia, N., Montserrat, L., Dominguez, L., Zorn, G. B. (2009). Multiresistance in Pasteurella multocida is mediated by coexistence of small plasmids. Antimicrob Agents Chemother 53:3399-3404. http://dx.doi.org/10.1128/AAC.01522-08.

[20] Shivachandra, S. B., Kumar, A. A., Biswas, A., Ramakrishnan, M. A., Singh, V. P. and Srivastava, S. K. (2004). Antibiotic sensitivity patterns among India strains of avian Pasteurella multocida. Trop. Anim.Health Product. 36: 743-750. http://dx.doi.org/10.1023/B:TROP.0000045950.35070.7f

[21] White, D. G., Zhao, S., Simjee, S., Wagner, D. D. and McDermott, P .F. (2002). Antimicrobial resistance of foodborne pathogens. Microbial. Infect. 4: 405-412. http://dx.doi.org/10.1016/S12864579(02)01554-X. 